\title{
ARTICLE
}

\section{Phase control for quasi-2D blue emitters by spacer cation engineering}

Received 00th January 20xx Accepted 00th January 20xx DOI: $10.1039 / \times 0 \times x 00000 x$

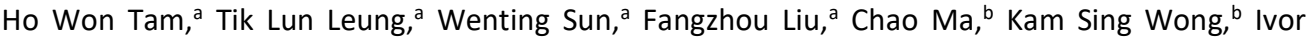 \\ Lončarić, ${ }^{c}$ Luca Grisanti, ${ }^{c}$ Juraj Ovčar, ${ }^{c}$ Željko Skoko, ${ }^{d}$ Jasminka Popović*e and Aleksandra B. \\ Djurišić*a
}

\section{Introduction}

Metal halide perovskites are of significant interest for light emitting applications due to their high colour purity and consequently wide colour gamut, potential for high luminescence quantum yields, high carrier mobilities, and the low temperature, low cost deposition. ${ }^{1}$ Perovskite LEDs (PeLEDs) have demonstrated rapid progress from the first reports in 2014 with efficiencies of the order $10^{-2} \%$ to over $20 \%$ at present for green, red, and near infrared PeLEDs. ${ }^{1,2}$ However, the efficiency of blue LEDs is lagging behind those achieved in other spectral regions..$^{2,3}$ Therefore, there is considerable interest in the development of novel perovskite blue emitters. ${ }^{3}$

Among different kinds of perovskite emitters, quasi-2D layered perovskites are of particular interest due to larger exciton binding energies and improved stability compared to commonly used 3D perovskite materials. ${ }^{1,4,5}$ Consequently, these materials have been extensively studied. ${ }^{1-35}$ The Ruddlesden-Popper (RP) quasi-2D perovskites have a formula

\footnotetext{
a. Department of Physics, The University of Hong Kong, Pokfulam, Hong Kong SAR. E-mail:dalek@hku.hk

b. Department of Physics, The Hong Kong University of Science and Technology, Clearwater Bay, Hong Kong.

c. Division of Theoretical Physics, Ruđer Bošković Institute, Zagreb, Croatia.

d. Department of Physics, Faculty of Science, University of Zagreb.

e. Division of Materials Physics, Ruđer Bošković Institute, Zagreb, Croatia. E-mail: Jasminka.Popovic@irb.hr.

†Electronic Supplementary Information (ESI) available: See
} DOI: $10.1039 / x 0 \times x 00000 x$
$A^{\prime}{ }_{2} A_{n-1} B_{n} X_{3 n+1}$ where $A^{\prime}$ is the bulky spacer cation, $A$ is a small organic cation or $\mathrm{Cs}^{+}, \mathrm{B}$ is divalent metal cation (commonly $\mathrm{Pb}^{2+}$ or $\left.\mathrm{Sn}^{2+}\right)$, and $\mathrm{X}$ is a halide anion, while $n$ is the number of layers of $\mathrm{BX}_{6}$ octahedra separated by a bilayer of $A^{\prime}$ spacer cations. ${ }^{1,3}$ As $n$ decreases, exciton binding energy and bandgap increase, while for $n=\infty$ 3D perovskite $\mathrm{ABX}_{3}$ is obtained. ${ }^{1,3}$ Therefore, the emission properties can be modified by adjusting $n$, which is of significant interest to avoid issues related to halide de-mixing during operation which leads to spectral in-stabilities in LEDs using mixed halide perovskite emitters. ${ }^{2,23}$

However, while the concept of band gap tuning via the selection of $n$ works perfectly in the case of exfoliated single crystals, in the case of thin films it is not so straightforward, since thin films of RP perovskites typically contain multiple $n$ phases. ${ }^{4,5,8,15,19,26}$ Due to the presence of multiple phases and the funneling phenomenon, ${ }^{13,15,18,22,26}$ the films typically exhibit emission from the lowest band gap phase present, i.e. the largest $n$. As a result, even films that mainly consist of low $n$ numbers ( $n=2$ or $n=3$ ) tend to exhibit green emission centered at wavelengths over $500 \mathrm{~nm}$ instead of the blue emission. ${ }^{6,13,22,26}$ Hence, the achievement of blue emission can require the use of mixed halide anions, ${ }^{22}$ which defeats the purpose of the emission tuning by adjusting the number of layers $n$ to avoid spectral instabilities related to ion migration induced halide segregation. ${ }^{24}$ In addition, increased phase purity is desirable to improve spectral stability. ${ }^{29}$ Consequently, full control over the phase composition of the films is essential for the achievement of emission in desired wavelength range..$^{5-8,14,19-21}$ Despite all the attempts to achieve 
phase purity for some commonly used spacer cations, such as phenylethylammonium (PEA), it is well established that along with the formation of desired $n=2$ phase, the crystallization of $n=1$ phase often cannot be avoided. ${ }^{8,19,21}$ For the PEA-based films prepared from stoichiometric $n=2$ solutions, the formation of $n=1$ phase also implies the unavoidable formation of higher $n$ phases, which commonly results in efficient energy transfer to higher $n$ phases ${ }^{19,21}$ and green emission instead of desired blue or sky-blue emission.

To achieve high $n=2$ phase purity that would ensure blue emission, the choice of precursors, the excess amount of ligand, additives (molecules or nanoparticles), the utilization of mixed small cations, or mixed spacer cations have been investigated, $2,3,7,8,16,19,21,23$ and the improvements in the achieved emission spectra have been demonstrated. Among mentioned approaches aiming to achieve of high phase purity for low $n$ numbers, one additional pathway, namely, the diversity of spacer cation structural features on the resulting properties represents the good alternative. Unfortunately, the relationship between the structure of spacer cation and the resulting film properties is still poorly understood, and moreover the systematic investigations on the effects of spacer cation choice on the phase purity, and consequently optical properties, have been scarce. ${ }^{36-39}$

We have previously shown that high $n=2$ phase purity films emitting pure blue emission could be obtained by the utilization butylammonium (BA) instead of PEA. ${ }^{21}$ This can be attributed to strong interaction between PEA cations due to $\pi$ $\pi$ stacking ${ }^{34}$ which favours the formation of $n=1$ phase. ${ }^{19,21}$ Unfortunately, alkyl chain spacer cations result in perovskite films with inferior environmental stability ${ }^{21}$ and lower light emission intensity compared to phenyl-ring containing spacers, such as PEA. ${ }^{21,33}$ This can be attributed to the differences in crystal rigidity and electron-phonon coupling, ${ }^{33}$ which are responsible for a significant photoluminescence quantum yield difference between $\mathrm{PEA}_{2} \mathrm{PbBr}_{4}$ and $\mathrm{BA}_{2} \mathrm{PbBr}_{4}$ (79\% vs. $17 \%$ ). ${ }^{33}$ However, using mixed $B A, P E A$ spacer cations allowed the formation of perovskite films combining favourable properties of both BA and PEA and achieve brighter emission and improved environmental stability while maintaining phase purity. ${ }^{21}$ Thus, here we investigate the possibility of achievement of phase purity via rational modifications of spacer cation, instead of physical mixing of different cations. Knowing that the perovskites with phenyl ring-containing spacer cations typically exhibit bright emission but poor phase purity while alkyl chain-based spacer cations exhibit improved phase purity but less efficient emission, ${ }^{19,21,33}$ we have conducted a systematic study that investigates the utilization of spacer cations containing both the aromatic ring and different alkylammonium moieties in order to gain an insight on the mechanisms governing the film properties. Therefore, thin films containing spacer cations with a phenyl ring and alkyl chains of different length, namely 1 carbon atom (benzylammonium, BZA), 2 carbon atoms (PEA), and 3 carbon atoms (phenylpropylammonium, PPA) have prepared. The structure diagrams of spacer cations are shown in Fig. S1+, Supporting Information. These spacer cations have been previously investigated for solar cell applications, and while the length of alkyl chain was found to affect the conductivity and photovoltaic performance, the effects on phase purity for low $n$ numbers and LED performance were not investigated.

Our work demonstrates that the proportion of $n=1$ impurity phase decreases as the length of the aliphatic chain of spacer cation increases and this trend holds for both methylammonium (MA) and formamidinium (FA) containing films. Consequently, we achieved high phase purity $n=2$ films containing PPA cations and obtained sky blue emitting LEDs with emission peak centered at $\sim 480 \mathrm{~nm}$ with the best
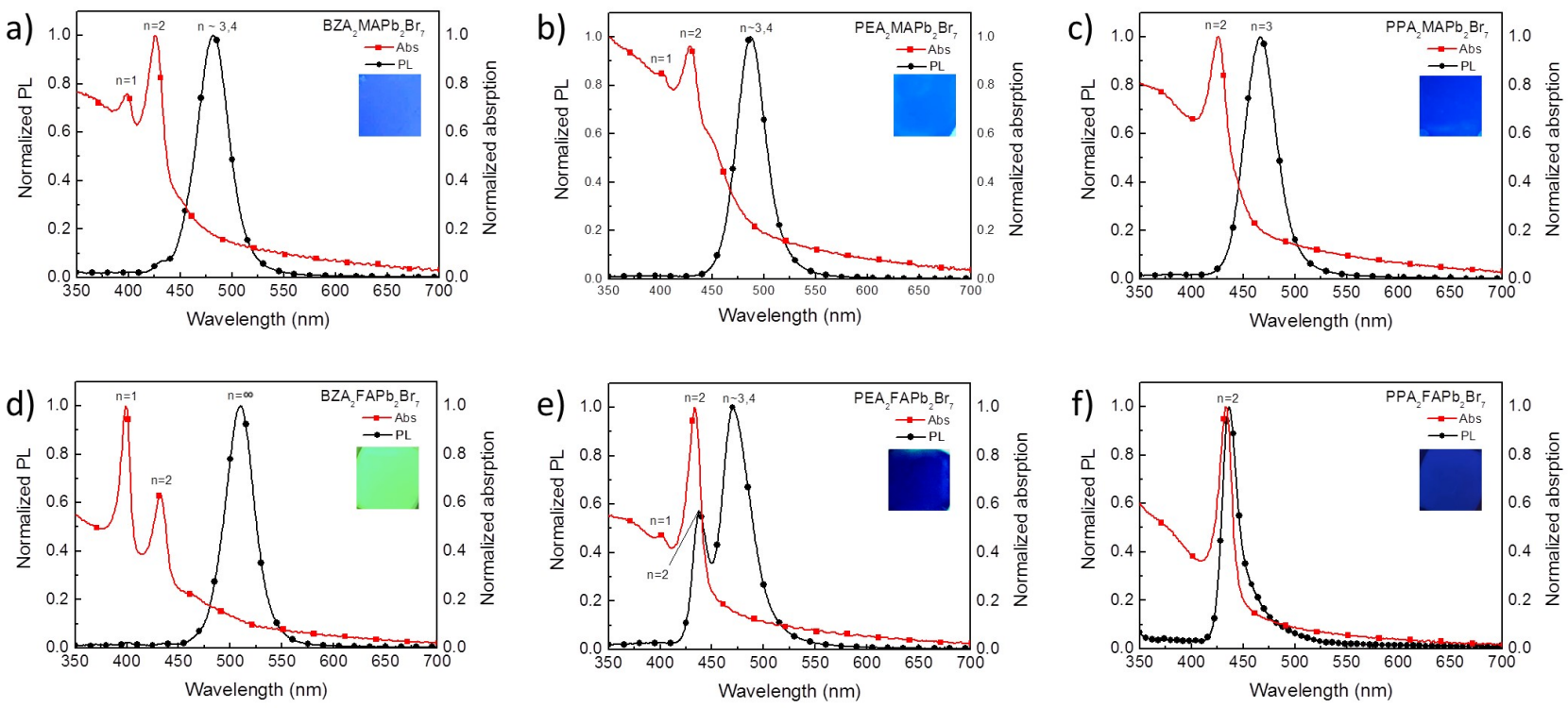

Fig. 1 Absorption and PL spectra of a) $\mathrm{BZA}_{2} \mathrm{MAPb}_{2} \mathrm{Br}_{7}$, b) $\left.\mathrm{PEA}_{2} \mathrm{MAPb}_{2} \mathrm{Br}_{7}, \mathrm{c}\right) \mathrm{PPA}_{2} \mathrm{MAPb}_{2} \mathrm{Br}_{7}$, d) $\mathrm{BZA}_{2} \mathrm{FAPb}_{2} \mathrm{Br}_{7}$, e) $\mathrm{PEA}_{2} \mathrm{FAPb}_{2} \mathrm{Br}_{7} \mathrm{and} \mathrm{f}_{\mathrm{f}} \mathrm{PPA}_{2} \mathrm{FAPb}_{2} \mathrm{Br}_{7}$. The insets show corresponding photos of the films under UV illumination. 
maximum external quantum efficiency (EQE) of 3.35\%. The reasons for superior performance of PPA spacer cation are discussed in the following.

\section{Results and discussion}

Fig. 1 shows the absorption and PL spectra of MA- and FAbased perovskite films prepared with BZA, PEA, and PPA spacer cations corresponding to stoichiometry of $n=2$. As expected based on common observations in quasi-2D RP perovskites, due to energy funneling phenomenon PL spectra can contain red-shifted peaks compared to absorption, indicating the presence of higher $n$ phases at low enough content so that their presence is not necessarily observable in the absorption spectra and/or XRD patterns. ${ }^{25,26}$ The peaks in absorption and PL spectra in RP materials are located at similar positions for different spacer cations, ${ }^{19,25-27}$ while the peak shape can indicate the presence of multiple $n$ phases, i.e. larger linewidth and symmetric lineshape are typically observed when contributions from different individual $n$ phases could not be resolved. In contrast, single-phase samples such as single crystals and exfoliated flakes typically exhibit narrow asymmetric photoluminescence, with the exfoliated samples exhibiting more narrow emission with lower emission tail due to their improved crystal quality and reduced defects. ${ }^{27}$ In the MA series, we can clearly resolve absorption peaks corresponding to $n=1(\sim 400 \mathrm{~nm})$ and $n=2$ $(\sim 430 \mathrm{~nm})$ for $\mathrm{BZA}_{2} \mathrm{MAPb}_{2} \mathrm{Br}_{7}$, while the emission is located at $\sim 480 \mathrm{~nm}$ (likely corresponding to $n=4$ ), with a small shoulder corresponding to $n=2$ emission. In the case of $\mathrm{PEA}_{2} \mathrm{MAPb}_{2} \mathrm{Br}_{7}$, the contribution of $n=1$ phase in the absorption spectrum diminishes, but there is a shoulder corresponding to $n=3$ phase at $\sim 450 \mathrm{~nm}$. In the emission spectra, single broad peak centered at $\sim 487 \mathrm{~nm}$ is observed, which likely corresponds to $n=4$ with possible contributions of $n=5$ phase. Unlike the cases of BZA and PEA where $n=1$ absorption peaks are clearly present, in the absorption spectra of $\mathrm{PPA}_{2} \mathrm{MAPb}_{2} \mathrm{Br}_{7}$ we can only resolve the peak corresponding to $n=2$. Single emission peak is centered at $\sim 466 \mathrm{~nm}$ and likely corresponds to a small amount of $n=3$ phase that is not detectable in the absorption spectra. This phenomenon can be commonly observed in quasi-2D perovskites. For example, $\mathrm{PEA}_{2}\left(\mathrm{Rb}_{0.6} \mathrm{Cs}_{0.4}\right)_{\mathrm{n}-1} \mathrm{~Pb}_{n} \mathrm{Br}_{3 n+1}$ samples with $n=2$ stoichiometry exhibited PL from $n=3$ phase only, while the absorption spectrum was dominated by $n=2$ phase peak. ${ }^{23}$ The presence of multiple phases in quasi-2D perovskites and the existence of energy funneling commonly results in a significant redshift of the emission peaks due to emission occurring from a small fraction of higher $n$ phase. This shift is larger than the Stokes' shift corresponding to the difference in peak position of the absorption and emission peak corresponding to the same phase that can be observed in samples which do not contain higher $n$ phases, such as quantum dots, and rarely thin films for example $\mathrm{PPA}_{2} \mathrm{FAPB}_{2} \mathrm{Br}_{7}$ (Fig. 1f) and $\mathrm{BA}_{2} \mathrm{MAPB}_{2} \mathrm{Br}_{7} .{ }^{21}$ Some reported examples of red- a)

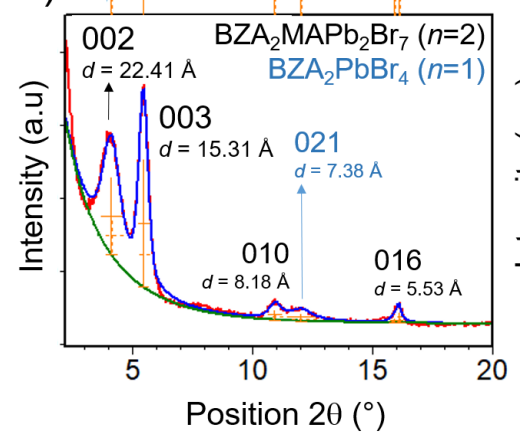

d)

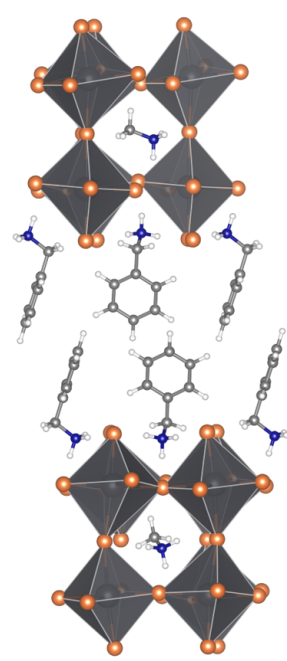

$\mathrm{BZA}_{2} \mathrm{MAPb}_{2} \mathrm{Br}_{7}$ b)

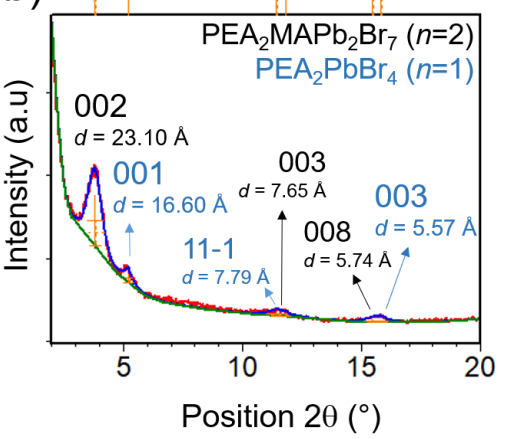

Position $20\left(^{\circ}\right)$

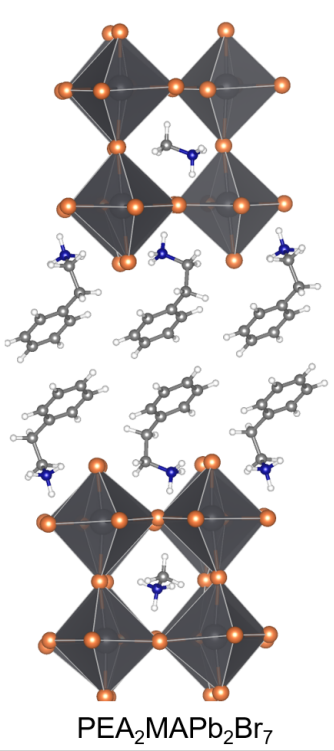

c)
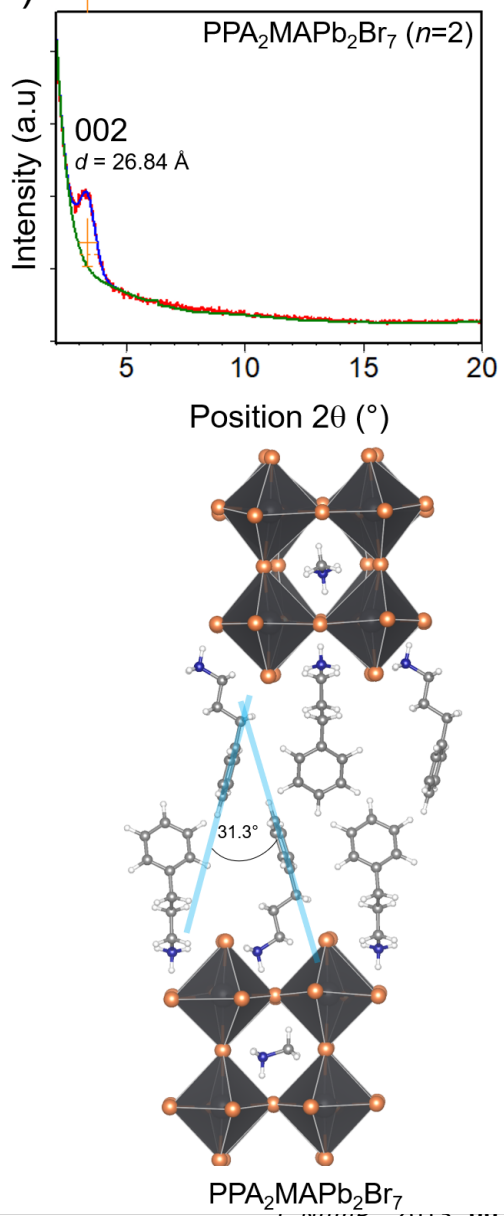
13 Fig. 2 Le Bail structural refinement on the XRD data of a) $\mathrm{BZA}_{2} \mathrm{MAPb}_{2} \mathrm{Br}_{7}$, b) $\left.\mathrm{PEA}_{2} \mathrm{MAPb}_{2} \mathrm{Br}_{7}, \mathrm{c}\right) \mathrm{PPA}_{2} \mathrm{MAPb}_{2} \mathrm{Br}_{7}$. Panel d) shows the perovskite structures with $\mathrm{BZA}$, $\mathrm{PEA}$ and $\mathrm{PPA}$ cations, respectively. 
shift of emission in different RP perovskites due to energy funneling to higher $n$ phase are summarized in Table $\mathrm{S}^{+}{ }^{\text {, }}$ Supporting Information. Thus, the fact that observed emission peak corresponds to the highest $n$ phase with negligible contributions of lower $n$ phases which are clearly observable in the absorption spectra indicates that funneling process in MAbased films is very efficient.

In the FA series, we can also observe similar trends in terms of diminishing proportion of $n=1$ phase as the length of the alkyl chain in spacer cation increases. However, in this case funneling is not as efficient as in the MA, as evidenced from the presence of $\mathrm{PL}$ emission peaks corresponding to lower $n$ phases indicating incomplete energy transfer from lower $n$ to higher $n$ phases in the samples exhibiting multiple phases in the absorption spectra. We observe multiple peaks in the emissions of $\mathrm{BZA}_{2} \mathrm{FAPb}_{2} \mathrm{Br}_{7}$ and $\mathrm{PEA}_{2} \mathrm{FAPb}_{2} \mathrm{Br}_{7}$, while for $\mathrm{PPA}_{2} \mathrm{FAPb}_{2} \mathrm{Br}_{7}$ a single emission peak at $\sim 436 \mathrm{~nm}$ is observed which corresponds to $n=2$ in agreement with the observed absorption spectrum. The shape of the emission peak resembles that observed in single-phase exfoliated flakes, ${ }^{27}$ consistent with high phase purity of $n=2$ sample. Since the choice of the small cation can affect the crystallization of the samples and thus the distribution of different phases, this likely contributes to significant differences in funneling observed for MA and FA series, since funneling is dependent on the average distance between low $n$ and high $n$ domains, ${ }^{18}$ as well as the prevalence of higher $n$ domains. ${ }^{15,18}$ The distance between domains and the concentration of higher $n$ domains are dependent on the film compositional distribution, ${ }^{18}$ while the effects of distance and the dynamics of the process are dependent on the excited species (excitons vs. free carriers). ${ }^{35}$

Introducing the various modifications of spacer cations have been explored in order to tune phase distribution. It has been reported that the change in intermolecular interactions such as hydrogen bonding, van der Waals interaction, arylperfluoroaryl and $\mathrm{CH} \cdots \pi$ interactions between organic cations affects the phase distribution, surface morphology and crystal orientation. ${ }^{19,36,38}$ Also, it has been shown that even a small change in the structure of spacer cation, such as monofluorination, affects the packing arrangements of the organic cations and their orientational disorder. ${ }^{39}$ In addition to the above mentioned modifications, absorption spectra of both MA- and FA-based perovskites (Fig. 1) suggest that the length of the alkyl chain in the spacer cations also plays a significant role in the stabilization of targeted $n=2$ phase In particular, as the length of alkyl chain increases (from one carbon atom in the case of BZA to three carbon atoms in the case of PPA), electron withdrawing effect of the nitrogen atom from the aromatic ring becomes less and less efficient. That, in turn, might allow for observed stabilization of $n=2$ phase in the case of PPA due to the formation of stronger interactions between perovskite layer and organic bilayer compared to the shorter BZA and PEA cations.

Perovskite films with BZA, PEA and PPA cations have also been investigated by the powder X-ray diffraction (PXRD). As it can be seen from Fig. 2, the increase of spacer cation length is accompanied with the shift of $00 /$ reflection towards the lower
$2 \theta$ angles indicating the increment of bilayer thickness. Similar trend to MA series has also been observed in the case of FAbased films (Fig. S2 + ), as expected. Since $n=2$ structures of RP perovskites with BZA and PPA spacer cations have not been the experimentally determined and reported so far, synergic approach between PXRD structural analysis and computational calculations utilizing the DFT has been undertaken in order to gain an insight into the structural features and packing arrangements for each spacer cation. Our previous work showed that minimum energy structure of $\mathrm{PEA}_{2} \mathrm{MAPb}_{2} \mathrm{Br}_{7}$ consist of off-centered parallel PEA cations within the spacer bilayer. ${ }^{21}$ Fig. $2 b$ shows Le Bail refinement for $\mathrm{PEA}_{2} \mathrm{MAPb}_{2} \mathrm{Br}_{7}$ converged with refined cell $a=8.31(7) \AA$, $b=8.12(5) \AA$, $c=46.11(5) \AA, \alpha=89.74(1)^{\circ}, \quad \beta=94.74(2)^{\circ}$ and $\gamma=90.26(1)^{\circ}$. In addition to the reflections belonging to $n=2$, XRD patterns for also show diffraction lines that belong to the $n=1 \mathrm{PEA}_{2} \mathrm{PbBr}_{4}$ phase. For the Le Bail refinement the iodide analogue $\mathrm{PEA}_{2} \mathrm{Pbl}_{4}$ lattice ${ }^{40}$ has been used. The refinement for $\mathrm{PEA}_{2} \mathrm{PbBr}_{4}$ converged with $c=17.41(2) \AA$ which is slightly smaller compared to iodine analogue $17.69 \AA$, as expected due to the smaller radii of $\mathrm{Br}$.

In the course of structural relaxation calculation for case of $\mathrm{BZA}_{2} \mathrm{MAPb}_{2} \mathrm{Br}_{7}$, as a starting model we have considered both the arrangement as reported for $n=1 \quad \mathrm{BZA}_{2} \mathrm{Pbl}_{4}{ }^{41}$ and the parallel offset stacking arrangement similar to one reported for PEA. ${ }^{42}$ Difference between those models relates to the way phenyl moieties are mutually assembled within one layer. In the structure of $\mathrm{BZA}_{2} \mathrm{Pbl}_{4}$, phenyl rings of $\mathrm{BZA}$ cations are assembled in T-shaped fashion, while in the second model rings within each layer were mutually parallel. Structural relaxation of the latter model yielded a minimum energy structure. Minimum energy structure, as derived from DFT calculations, have been used in Le Bail refinement on PXRD data (Fig. 2a); good agreement suggests that $\mathrm{BZA}_{2} \mathrm{MAPb}_{2} \mathrm{Br}_{7}$ crystallizes with refined lattice-parameters $a=7.92(6) \AA$, $b=8.18(4) \AA, \quad c=47.20(1) \AA, \alpha=90.01(1)^{\circ}, \quad b=107.00(1)^{\circ}$ and $\gamma=90.14(1)^{\circ}$. Again, as in the case of PEA-based films, diffraction lines that belong to the $n=1 \quad \mathrm{BZA}_{2} \mathrm{PbBr}_{4}$ can be observed in PXRD pattern. The $n=1$ structure exhibits the longest cell periodicity of 27.2(1) that is smaller, as anticipated, compared to its iodine analogue $\mathrm{BZA}_{2} \mathrm{Pbl}_{4}$ reported by Papavassiliou et al. ${ }^{41}$

The minimum energy structures for $n=2$ BZA and PEAbased perovskites are shown in Fig. $2 \mathrm{~d}$ and it is essential to notice that the bilayer in both structures is characterized by parallel assembly (orientation) of phenyl rings. Interestingly, in the case of $\mathrm{PPA}_{2} \mathrm{MAPb}_{2} \mathrm{Br}_{7}$ computational relaxation favours quite different assembly of PPA cations compared to the shorter BZA and PEA cations, with the non-parallel arrangement of aromatic rings corresponding to the minimum energy structure. The angle between planes in which phenyl rings lay amounts to $31.3^{\circ}$. The lattice-parameters ( $a=8.177 \AA$, $b=8.173 \AA \AA, c=54.378 \AA, \alpha=89.72^{\circ}, \quad b=90.41^{\circ}$ and $\gamma=90.18$ ) obtained from the computation relaxation are in the good agreement with diffraction reflection 002 at $d=26.84 \AA$. The observed change in the way aromatic rings are arranged in the structure of PPA compared to the BZA and PEA is caused by 
the different interplay of intermolecular interactions, and is likely to have a significant impact on the phase distribution. ${ }^{19,34,36}$ The parallel arrangement of the aromatic rings for BZA and PEA is a result of strong van der Waals interactions that consequently favors the formation of $n=1$ phase. ${ }^{19}$ In contrast, non-parallel arrangement of aromatic rings for PPA, corresponds to weaker the $\pi-\pi$ interaction and enhanced $\mathrm{CH} \cdots \pi$ interactions, ${ }^{36}$ which could suppress the formation of the $n=1$ phase. Structural features of minimum energy structures for $n=2$ BZA, PEA and PPA-based perovskites, as obtained by the computational relaxation, are given in Table $\mathrm{S} 2+$, Supporting Information.
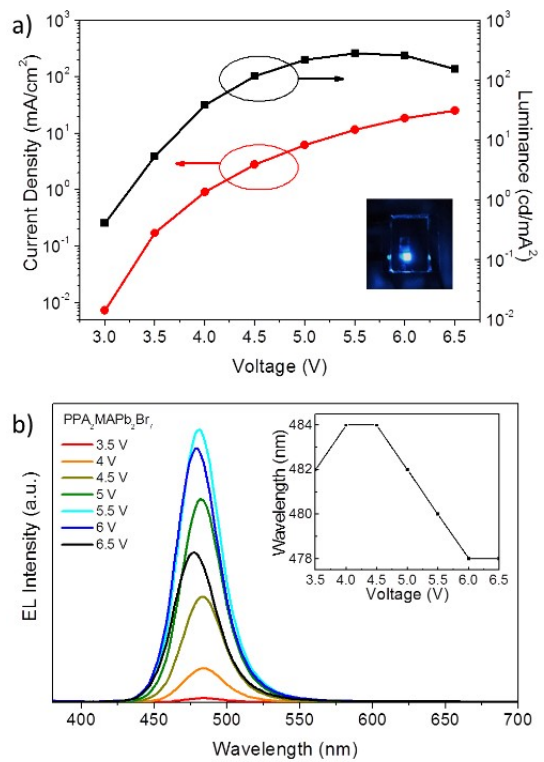

Figure 3 a) L-I-V plots (device photo is shown in the inset) and b) EL spectra vs. bias voltage for $\mathrm{PPA}_{2} \mathrm{MAPb}_{2} \mathrm{Br}_{7}$ devices. The center wavelength position as a function of bias voltage is shown in the inset.

Consequently, due to the enhanced phase purity and prominent blue emission, PPA-based perovskites are of interest for applications in blue LEDs. Since our target is to obtain the emission in the blue spectral range, we will focus on the samples prepared with $n=2$ solution stoichiometry. For samples prepared with $n=3$, presence of higher $n$ phases results in the red shift of the emission, as shown in Fig. S3+, Supporting information. While the phase composition can be tuned to some degree by deposition conditions, for $n>2$ solution stoichiometry there is a significant emission contribution in the green spectral range. The device architecture and SEM image of the cross-section are shown in Fig. S4t, Supporting information. SEM images and XRD patterns of $\mathrm{PPA}_{2} \mathrm{MAPb}_{2} \mathrm{Br}_{7}$ and $\mathrm{PPA}_{2} \mathrm{FAPb}_{2} \mathrm{Br}_{7}$ films are shown in Figs. $\mathrm{S} 5$ and $\mathrm{S} 6+$, Supporting Information, respectively. From SEM images it can be observed that the film morphology is better for the MA-based films (fewer pinholes), but XRD patterns point to the higher crystallinity in the case of FAbased films. Higher crystallinity of FA-based films on indium tin oxide (ITO) $/ \mathrm{NiO}_{x} / \mathrm{PVK}$ is in agreement with the trends previously observed on ITO substrates (Fig. $2 \mathrm{c}$ and S2+). Since MA-based films exhibited better morphology while FA-based films exhibited crystallinity, we proceeded with the device fabrication for both types of small organic cations.

Fig. 3 shows the $L-I-V$ curves of the $\mathrm{PPA}_{2} \mathrm{MAPb}_{2} \mathrm{Br}_{7}$ champion device and EL spectra as a function of bias voltage. The results of a stability test of encapsulated devices in ambient at constant bias voltage of $4 \mathrm{~V}$ are shown in Fig. S7+, Supporting Information and a $\mathrm{T}_{50}$ of $\sim 52 \mathrm{~s}$ is obtained. The devices exhibit sky-blue emission. While small spectral shifts can be observed, all the peak centers are within $481 \pm 3 \mathrm{~nm}$ spectral range and thus within sky-blue spectral range. Furthermore, with increasing bias voltage we observe a small blue shift of the emission peak, which is significantly different from commonly observed red shift with increasing bias or increasing operating time that has been attributed to Ostwald ripening or increases in grain/particle size, ${ }^{23,24}$ as well as electrostatic effects and ion migration (the later is applicable to mixed halide devices). ${ }^{24}$ To establish whether bias resulted in any lasting changes in the sample composition, we measured the PL spectra in the device area and outside device area for different bias voltages shown in Fig. $\mathrm{S}^{+}{ }^{+}$, Supporting Information. We can observe that increasing bias results in a small blue shift of the $\mathrm{PL}$, confirming that there is a permanent change in the film properties with increasing bias. The change can likely be attributed to small changes in sample crystal structure, i.e. contributions of different $n$ phases to the emission. Changes in crystallinity could occur due to recrystallization due to Joule heating during bias. ${ }^{23}$ Since the peak shift is very small, any phase composition changes are too small to be detectable by other techniques, such as XRD, since PL is typically most sensitive to the changes in phase composition of the film. Small red shift of $\sim 2 \mathrm{~nm}$ in the $\mathrm{EL}$ emission at low bias likely originates from electrostatic effects, ${ }^{24}$ consistent with small magnitude of the peak shift and the fact that it does not appear in PL spectra after bias which only show blue shift. The EL spectra are red-shifted compared to PL spectra, similar to other reports on quasi-2D perovskite LEDs, ${ }^{19,26,30-32}$ although in some cases no shifts were observed.23,31 Such red-shift is typically an indication of emission from higher $n$ phase, ${ }^{19}$ while the differences reported in the literature could occur due to the differences in the sample composition, as well as differences in the mechanisms responsible for the funneling (Förster-type exciton transfer, as well as free-carrier charge transfer), ${ }^{35}$ which can result in differences in the overall effect of the electric field on the peak position. In general, excited species in PL are excitons (for low $n$ phases which have high exciton binding energy), while in EL electrons and holes are injected from the opposite electrodes and they recombine in the active layer. In $\mathrm{PL}$, carrier transfer between different phases occurs due to energy level differences at interfaces, while in EL injected carriers are subjected to the external electric field which can enhance the funneling process and result in the majority of charges recombining in the smallest bandgap pahse and consequently red shift of the emission if there are higher $\mathrm{n}$ phases present. ${ }^{26}$ In addition, differences in the excitation process between EL 
and PL can result in different carrier densities, which in turn would affect the light emission. ${ }^{26}$

Fig. 4 shows the EQE dependence on the bias, and illustrates the device reproducibility. The obtained devices exhibit an average EQE of $2.54 \pm 0.45 \%$ ( 26 devices). The best performing device exhibits a maximum EQE of $3.35 \%$ and a maximum luminance of $286 \mathrm{~cd} / \mathrm{m}^{2}$ (peak centered at $480 \mathrm{~nm}$ ). This is significantly higher than that obtained for $\mathrm{PPA}_{2} \mathrm{FAPb}_{2} \mathrm{Br}_{7}$ devices, which exhibits an average EQE of $0.07 \pm 0.01 \%$ (7 devices), with corresponding EL spectra and EQE histogram shown in Fig. S9t, Supporting Information. Observed LED performance difference for MA- and FA-based perovskites is in agreement with significantly higher photoluminescence quantum yield (PLQY) of $2.1 \%$ determined for $\mathrm{PPA}_{2} \mathrm{MAPb}_{2} \mathrm{Br}_{7}$ films with trioctylphosphine oxide (TOPO) ${ }^{26}$ as a passivating agent, compared to $0.16 \%$ obtained for $\mathrm{PPA}_{2} \mathrm{FAPb}_{2} \mathrm{Br}_{7}$ films with TOPO. The differences between PLQY and EQE values are likely due to the fact that EQE is measured on a multilayer structure which affects the light extraction and the fact that the substrate on which the perovskite film is deposited affects its quality. ${ }^{17}$ Due to the fact that perovskite LED structure contains multiple layers which emit light under optical excitation (PVK, TPBi), it is not possible to directly measure PLQY of the perovskite film contained within the device. Furthermore, some differences in measured PLQY and EQE can occur due to differences in achieved charge carrier densities in the emitting layer by optical excitation and electrical injection.

The obtained efficiency is significantly higher than that reported for PPA-Cs perovskite, where in all cases for different PPA:Cs ratios green emission was obtained and the highest efficiency achieved was $0.01 \%{ }^{12}$ It should also be noted that FA-based devices exhibit multiple emission peaks consistent with the emission corresponding to $n=2$ phase and a large $n$ or possibly 3D phase, while in MA-based devices we observe emission corresponding to $n=3$ phase, as illustrated in Fig. S10t, Supporting Information. The change in the optical properties for FA-based films compared to those deposited on ITO likely occurred due to differences in crystallization of the films on $\mathrm{NiO}_{x} / \mathrm{PVK}$, since the film quality of the perovskite is strongly dependent on the underlying material. ${ }^{17}$ While the presence of additional phases is too small to be detectable from the comparison of XRD patterns of films on ITO and ITO/NiO $/$ PVK) (Fig. 2c, Fig. S2 ${ }^{+}$and Fig. S6 ${ }^{+}$, Supporting Information), the presence of different $n$ phases is very clear from the absorption and PL spectra. From the obtained absorption and PL spectra in Fig. S10 + , Supporting Information we can conclude that in MA-based films efficient funneling occurs from dominant $n=2$ to the small amount of $n=3$ and 4 phase, leading to efficient sky-blue device performance. In contrast, there is no efficient funneling from $n=2$ phase to green emitting higher $n / 3 \mathrm{D}$ phase in FA-based films, resulting in multiple emission peaks and low efficiency. It is known that the non-optimized energy landscape, which lacks suitable distribution of different bandgap energy domains that can facilitate energy transfer, leads to significantly lower emission efficiency. ${ }^{15}$ Since the funneling is dependent not only on the concentrations of phases with higher $n$ but also on the distance between them, ${ }^{18}$ increased crystallinity of FA-based films could result in increased distances between the lower $n$ and higher $n$ phases and consequently incomplete energy transfer.

Thus, likely reason for the inferior performance of FAbased devices is non-optimized compositional $n$ number distribution, preventing efficient energy transfer from the dominant wide bandgap $n=2$ phase to a small amount of high $n$ or 3D impurity phase (which cannot be resolved in XRD, but could explain small tail in the luminescence). In both absorption and XRD spectra, only $n=2$ phase can be resolved, while in the PL spectra we can observe the peaks corresponding to $n=2, n=3$, and a higher $n(\geq 8)$, showing clear lack of intermediate phases between $n=3$ and higher $n$, which would impede efficient energy transfer to high $n$ phase. Thus, despite lower crystallinity $\mathrm{PPA}_{2} \mathrm{MAPb}_{2} \mathrm{Br}_{7}$ emitters result in superior performance of sky-blue LEDs, facilitated by favourable energy funneling from dominant $n=2$ phase to $n=3,4$ phases contributing to sky-blue emission. In contrast, devices with $\mathrm{BZA}_{2} \mathrm{MAPb}_{2} \mathrm{Br}_{7}$ and $\mathrm{PEA}_{2} \mathrm{MAPb}_{2} \mathrm{Br}_{7}$ emitters exhibit green light emission, with emission center located above $500 \mathrm{~nm}$, as shown in Fig. S11+, Supporting Information.
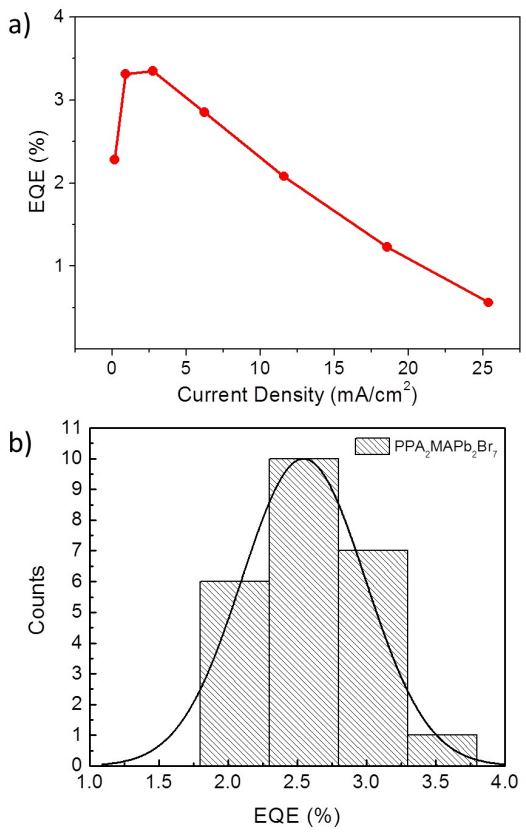

Fig. 4 a) EQE vs. current density and b) histogram plot illustrating reproducibility of the $\mathrm{PPA}_{2} \mathrm{MAPb}_{2} \mathrm{Br}_{7}$ devices (total of 26 devices).

\section{Experimental Section}

Materials

Phenylpropylammonium bromide (PPABr) and formamidinium Bromide $(\mathrm{FABr})$ were purchased from Xi'an Polymer Light Technology Corp. Benzylammonium bromide (BZABr), 
phenethylammonium bromide (PEABr), and methylammonium bromide (MABr) were purchased from Greatcell Solar Materials. Lead bromide $\left(\mathrm{PbBr}_{2}, 99.998 \%\right)$, acetonitrile (ACN, anhydrous, 99.8+\%), iso-propanol (IPA, anhydrous, 99.5+\%), $\mathrm{N}, \mathrm{N}$-dimethylformamide (DMF, anhydrous, 99.9\%) and trioctylphosphine oxide (TOPO, 98\%) were obtained from Alfa Aesar. Nickel(II) acetate tetrahydrate $\left(\mathrm{Ni}(\mathrm{Ac})_{2} \cdot 4 \mathrm{H}_{2} \mathrm{O}, 99.998 \%\right)$, chlorobenzene (anhydrous, 99.8\%), methyl acetate (MAc, anhydrous, 99.5\%), ethanolamine ( $\geq 99 \%)$ were purchased from Sigma Aldrich. Poly(9-vinylcarbazole) (PVK), 1,3,5-Tris(1phenyl-1Hbenzimidazol-2-yl)benzene (TPBi, >99.5\%) and cesium carbonate $\left(\mathrm{Cs}_{2} \mathrm{CO}_{3},>99.994 \%\right)$ were purchased from Luminescence Technology Corp. Hexafluorotetracyanonaphthoquinodimethane (F6TCNNQ, 99\%) was purchased from Shanghai Da Ran Chemicals. Aluminum pellets (Al, 99.999\%) were purchased from Kurt J. Lesker Company. All chemicals were used as received unless otherwise specified.

\section{Synthesis of quasi-2D perovskite thin films}

Perovskite precursor solution was prepared by dissolving the mixture of precursors according to corresponding stoichiometric ratio in DMF. For instance, precursor solution of $\mathrm{PPA}_{2} \mathrm{MAPb}_{2} \mathrm{Br}_{7}$ was obtained by dissolving $\mathrm{PbBr}_{2}(0.1 \mathrm{mmol}$, $36.7 \mathrm{mg}$ ), PPABr (0.1 mmol, $21.6 \mathrm{mg}$ ) and $\mathrm{MABr}(0.05 \mathrm{mmol}$, $5.6 \mathrm{mg}$ ) in $1 \mathrm{~mL} \mathrm{DMF}$, followed by stirring at room temperature. Perovskite films were then deposited on cleaned ITO glass substrates by spin-coating at $1000 \mathrm{rpm}$ for $10 \mathrm{~s}$ and then $4000 \mathrm{rpm}$ for $45 \mathrm{~s}$. $50 \mu \mathrm{L}$ of MAc antisolvent was added to the film after $5 \mathrm{~s}$ of spinning at $4000 \mathrm{rpm}$. The film was then vacuum dried for $30 \mathrm{~min}$ before any further treatment or characterization. All procedures were performed inside an argon filled glovebox.

\section{Characterization of quasi-2D perovskite film}

All optical measurements were performed with perovskite films encapsulated by quartz plates. Steady state PL spectra were measured by PDA-512 USB (Control Development Inc.) Fiberoptic Spectrometer using $\mathrm{He}-\mathrm{Cd}$ laser $(325 \mathrm{~nm}$ ) as the excitation source. Absorption spectra of the perovskite films were measured using Cary 50 UV-Vis spectrometer (Agilent Technologies). XRD patterns of the perovskite samples were obtained with Rigaku SmartLab 9kW X-ray Diffractometer with $\mathrm{Cu} \mathrm{K} \alpha$ radiation. Hitachi S-4800FEG Scanning Electron Microscope was used for morphology characterization of the perovskite films. External PLQY was estimated through a comparison technique of the encapsulated perovskite films to standard reference. PL of perovskite samples and the standard reference were measured using the same setup under the same conditions. He-Cd $325 \mathrm{~nm}$ continuous wave laser was used as the excitation source. Absorption of the samples are determined through evaluation of incident power, reflected power, and transmitted power of the laser, which were measured by a calibrated photodiode power meter from Newport. The PLQY of the perovskite films was then calculated by considering their PL intensity and absorption intensity ratio to those of standard reference, as described previously. ${ }^{21}$

\section{Device fabrication and characterization}

The LED structure was ITO/NiO $/$ F6TCNNQ/ PVK/ perovskite/TPBi/ $/ \mathrm{Cs}_{2} \mathrm{CO}_{3} / \mathrm{Al}$. Patterned ITO/glass substrates were cleaned sequentially using Decon 90, deionized water, acetone and ethanol, followed by oxygen plasma cleaning before use. $\mathrm{NiO}_{x}$ sol-gel solution was prepared by dissolving $\mathrm{Ni}(\mathrm{Ac})_{2} \cdot 4 \mathrm{H}_{2} \mathrm{O}(0.2 \mathrm{mmol})$ and ethanolamine $(0.2 \mathrm{mmol})$ in $1 \mathrm{~mL}$ of IPA and was stirred at $70{ }^{\circ} \mathrm{C}$ overnight. The $\mathrm{NiO}_{x}$ sol-gel solution was spin-coated on ITO/glass substrates at $3000 \mathrm{rpm}$ for $30 \mathrm{~s}$ followed by preheat treatment at $100{ }^{\circ} \mathrm{C}$ for $10 \mathrm{mins}$ and later annealing at $275^{\circ} \mathrm{C}$ for $1 \mathrm{hr}$ in air. F6TCNNQ solution (0.2 mg mL $\mathrm{mL}^{-1}$ in $\mathrm{ACN}$ ) was then spin-coated on $\mathrm{NiO}_{x}$ at 4000 rpm for $60 \mathrm{~s}$ inside argon-filled glovebox. $\mathrm{NiO}_{x} / \mathrm{F6TCNNQ}$ films were annealed overnight at $80{ }^{\circ} \mathrm{C}$. PVK solution $\left(5 \mathrm{mg} \mathrm{ml}^{-1}\right.$ in CB) was spin-coated on $\mathrm{NiO}_{x} / \mathrm{F} 6 \mathrm{TCNNQ}$ at $3000 \mathrm{rpm}$ for $30 \mathrm{~s}$ followed by annealing at $120{ }^{\circ} \mathrm{C}$ for 15 mins. Perovskite films were then deposited on $\mathrm{NiO}_{x} / \mathrm{F} 6 \mathrm{TCNNO} / \mathrm{PVK}$ layer as described above. TOPO solution ( $0.5 \mathrm{mg} \mathrm{ml}^{-1}$ in MAc) was further spincoated on perovskite layer for surface passivation. Finally, $\mathrm{TPBi}, \mathrm{Cs}_{2} \mathrm{CO}_{3}$ and $\mathrm{Al}$ were sequentially thermally evaporated through a shadow mask with thickness of $45 \mathrm{~nm}, 1 \mathrm{~nm}$, and $100 \mathrm{~nm}$, respectively, to complete the device. The active area defined by the mask was $0.04 \mathrm{~cm}^{2}$. The performance of the perovskite LEDs was characterized by electroluminescence (EL) measuring setup consisting of a programmable Keithley 2400 SourceMeter and a Photoresearch PR-670 spectroradiometer with SL-1X close-up lens. EL spectra were recorded by PR670 spectroradiometer.

\section{DFT calculations}

Density functional theory (DFT) calculations have been performed using the mixed Gaussian and plane-wave basis code $\mathrm{CP}_{2} \mathrm{~K}^{43}$ with the GTH-PBE pseudopotentials ${ }^{44-46}$ and $\mathrm{PBE}+\mathrm{D} 3^{47,48}$ exchange-correlation functional. We used the triple-zeta valence with two sets of polarization functions gaussian basis sets. ${ }^{49}$ The first Brillouin zone was sampled by the Monkhorst-Pack k-point mesh with density of $5 \AA$. The unit cell and the atoms were relaxed until the maximal force on any atom was smaller than $0.02 \mathrm{eV} / \AA$, and the pressure was smaller than $0.01 \mathrm{GPa}$.

\section{Conclusion}

This works investigates the opportunity to tune the optoelectronic properties of quasi-2D perovskites through a careful compositional engineering and demonstrates that the length of the alkylammonium tail of alkyl-phenyl-based spacer cations consisting of a phenyl ring and alkyl chain influences the arrangement of the spacer cations, which in turn significantly affect the phase distribution in the prepared films. The utilization of shorter alkyl chains with 1 and 2 carbon atoms results in the parallel assembly of phenyl rings within the organic bilayer which favors the formation of $n=1$ phase. 
Consequently, due to the change in solution stoichiometry, appearance of $n=1$ phase also additionally causes the formation of higher $n$ phases which result in undesirable emission wavelength shift into the green spectral region. On the other hand, when longer 3-carbon chains are introduced, a non-parallel assembly of phenyl rings arises that proves to be beneficial for the suppression $n=1$ phases. As a result of such an approach, films that exhibit blue emission are readily obtained, and sky blue LEDs with efficiency of $3.35 \%$ are demonstrated. Thus, our work explains how the structural characteristics of the spacer cation affects the crystallization and phase composition of the perovskite films which can facilitate design of spacer cations for efficient bromide-only blue emitting perovskite materials.

\section{Conflicts of interest}

There are no conflicts to declare.

\section{Acknowledgements}

This work was supported by the Seed Funding for Basic Research and Seed Funding for Strategic Interdisciplinary Research Scheme of the University of Hong Kong and Project PZS-2019-02-2068 financed by the "Research Cooperability" Program of the Croatian Science Foundation funded by the European Union from the European Social Fund under the Operational Programme Efficient Human Resources 20142020. The authors also acknowledge financial support from Shenzhen Science and Technology Commission Projects JCYJ20160530184523244 and JCYJ20170818141216288, European Union through the European Regional Development Fund - the Competitiveness and Cohesion Operational Programme (KK.01.1.1.06), and Croatian-Chinese bilateral project entitled "Quasi 2D organic-inorganic perovskites for light emitting applications"

\section{Notes and references}

1 M. Lu, Y. Zhang, S. Wang, J. Guo, W. W. Yu and A. L. Rogach, Adv. Funct. Mater., 2019, 29, 1902008.

2 N. K. Kumawat, X. K. Liu, D. Kabra and F. Gao, Nanoscale, 2019, 11, 2109.

3 C. H. A. Li, Z. Zhou, P. Vashishtha and J. E. Halpert, Chem. Mater., 2019, 31, 6003.

4 Z. Chen, Y. Guo, E. Wertz and J. Shi, Adv. Mater., 2019, 31, 1803514.

5 X. Gao, X. Zhang, W. Yin, H. Wang, Y. Hu, Q. Zhang, Z. Shi, V. L. Colvin, W. W. Yu and Y. Zhang, Adv. Sci., 2019, 20, 1900941.

6 H. D. Lee, H. Kim, H. Cho, W. Cha, Y. Hong, Y. H. Kim, A. Sadhanala, V. Venugopalan, J. S. Kim, J. W. Choi, C. L. Lee, D. Kim, H. Yang, R. H. Friend and T. W. Lee, Adv. Funct. Mater., 2019, 29, 1901225.

7 J. Qing, C. Kuang, H. Wang, Y. Wang, X. K. Liu, S. Bai, M. Li, T. C. Sum, Z. Hu, W. Zhang and F. Gao, Adv. Mater., 2019, 31, 1904243.

8 Y. Han, S. Park, C. Kim, M. Lee and I. Hwang, Nanoscale, 2019, 11, 3546.
9 P. Vashishtha, M. Ng, S. B. Shivarudraiah and J. E. Halpert, Chem. Mater., 2019, 31, 83.

10 B. Zhao, S. Bai, V. Kim, R. Lamboll, R. Shivanna, F. Auras, J. M. Richter, L. Yang, L. Dai, M. Alsari, X. J. She, L. Liang, J. Zhang, S. Lilliu, P. Gao, H. J. Snaith, J. Wang, N. C. Greenham, R. H. Friend and D. Di, Nat. Photonics, 2018, 12, 783.

11 R. Yang, R. Li, Y. Cao, Y. Wei, Y. Miao, W. L. Tan, X. Jiao, H. Chen, L. Zhang, Q. Chen, H. Zhang, W. Zou, Y. Wang, M. Yang, C. Yi, N. Wang, F. Gao, C. R. McNeill, T. Qin, J. Wang and W. Huang, Adv. Mater., 2018, 30, 1804771.

12 X. Xu, Z. Wang, J. Yu, L. Li and X. Yan, Nanoscale Res. Lett., 2019, 14, 255.

13 J. Yan, G. Croes, A. Fakharuddin, W. Song, P. Heremans, H. Chen and W. Qui, Adv. Opt. Mater., 2019, 7, 1900465.

14 H. Tsai, W. Nie, J. C. Blancon, C. C. Stoumpos, C. M. M. Soe, J. Yoo, J. Crochet, S. Tretiak, J. Even, A. Sadhanala, G. Azzellino, R. Brenes, P. M. Ajayan, V. Bulović, S. D. Stranks, R. H. Friend, M. G. Kanatzidis and A. D. Mohite, Adv. Mater., 2018, 30, 1704217.

15 L. N. Quan, Y. Zhao, F. P. G. de Arquer, R. Sabatini, G. Walters, O. Voznyy, R. Comin, Y. Li, J. Z. Fan, H. Tan, J. Pan, M. Yuan, O. M. Bakr, Z. Lu, D. H. Kim and E. H. Sargent, Nano Lett., 2017, 17, 3701.

16 Y. Zou, H. Xu, S. Li, T. Song, L. Kuai, S. Bai, F. Gao and B. Sun, ACS Photonics, 2019, 6, 1728.

17 Z. Ren, X. Xiao, R. Ma, H. Lin, K. Wang, X. W. Sun and W. C. H. Choy, Adv. Funct. Mater., 2019, 29, 1905339.

18 N. Yantara, A. Bruno, A. Iqbal, N. F. Jamaludin, C. Soci, S. Mhaisalkar and N. Mathews, Adv. Mater., 2018, 30, 1800818

19 J. Xing, Y. Zhao, M. Askerka, L. N. Quan, X. Gong, W. Zhao, J. Zhao, H. Tan, G. Long, L. Gao, Z. Yang, O. Voznyy, J. Tang, Z. H. Lu, Q. Xiong and E. H. Sargent, Nat. Commun., 2018, 9, 3541.

20 Y. Hu, L. M. Spies, D. Alonso-Álvarez, P. Mocherla, H. Jones, J. Hanisch, T. Bein, P. R. F. Barnes and P. Docampo, J. Mater. Chem. A, 2018, 6, 22215.

21 T. L. Leung, H. W. Tam, F. Z. Liu, J. Y. Lin, A. M. C. Ng, W. K. Chan, W. Chen, Z. B. He, I. Lončarić, L. Grisanti, C. Ma, K. S. Wong, Y. S. Lau, F. R. Zhu, Ž. Skoko, J. Popović and A. B. Djurišić, Adv. Opt. Mater., 2020, 8, 1901679.

22 Z. C. Li, Z. M. Chen, Y. C. Yang, Q. F. Xue, H. L. Yip and Y. Cao, Nat. Commun., 2019, 10, 1027.

23 Y. Z. Jiang, C. C. Qin, M. H. Cui, K. K. Liu, Y. M. Huang, M. H. Luo, L. Zhang, H. Y. Xu and S. S. Li, Nat. Commun., 2019, 10, 1868.

24 P. Vashishtha and J. E. Halpert, Chem. Mater. 2017, 29, 5965.

25 D. N. Congreve, M. C. Weidman, M. Seitz, W. Paritmongkol, N. S. Dahod, and W. A. Tisdale, ACS Photonics 2017, 4, 476.

26 X. L. Yang, X. W. Zhang, J. X. Deng, Z. Chu, Q. Jiang, J. H. Meng, P. Y. Wang, L. Q. Zhang, Z. G. Yin, J. B. You, Nat. Commun. 2018, 9, 570.

27 K. Leng, I. Abdelwahab, I. Verzhbitskiy, M. Telychko, L. Q. Chu, W. Fu, X. Chi, N. Guo, Z. H. Chen, Z. X. Chen, C. Zhang, Q. H. Xu, J. Lu, M. Chhowalla, G. Eda, K. P. Loh, Nat. Mater. 208, 17, 908.

28 W. Deng, X. C. Jin, Y. Lv, X. J. Zhang, X. H. Zhang, J. S. Jie, Adv. Funct. Mater. 2019, 29, 1903861.

29 H. Lin, J. Mao, M. C. Qin, Z. L. Song, W. J. Yin, X. H. Lu, W. C. H. Choy, Nanoscale 2019, 11, 16907.

30 S. Zeng, S. Shi, S. Wang and Y. Xiao, J. Mater. Chem. C 2020, 8, 1319.

31 S. Kumar, J. Jagielski, S. Yakunin, P. Rice, Y. Chiu, M. Wang, G. Nedelcu, Y. Kim, S. Lin, E. J. G. Santos, M. V. Kovalenko and C. Shih, ACS Nano 2016, 10, 9720.

32 S. Lee, D. Kim, I. Hamilton, M. Daboczi, Y. S. Nam, B. R. Lee, B. Zhao, C. H. Jang, R. H. Friend, J. Kim and M. H. Song, Adv. Sci. 2018, 5, 1801350. 
33 X. Gong, O. Voznyy, A. Jain, W. Liu, R. Sabatini, Z. Piontkowsky, G. Walters, G. Bappi, S. Nokhrin, O. Bushuyev, M. Yuan, R. Comin, D. McCamant, S. O. Kelley, E. H. Sargent, Nature Mater. 2018, 17, 550.

34 M. Y. Ban, Y. T. Zou, J. P. H. Rivett, Y. G. Yang, T. H. Thomas, Y. S. Tan, T. Song, X. Y. Gao, D. Credgington, F. Deschler, H. Sirringhaus, B. Q. Sun, Nat. Commun., 2018, 9, 3892.

35 A. H. Proppe, M. H. Elkins, O. Voznyy, R. D. Pensack, F. Zapata, L. V. Besteiro, L. N. Quan, R. Quintero-Bermudez, P. Todorovic, S. O. Kelley, A. O. Govorov, S. K. Gray, I. Infante, E. H. sergeant, G. D. Scholes, J. Phys. Chem. Lett. 2019, 10, 419.

36 L. Yan, J. Hu, N. Zhou, A. M. Moran and W. You, Sol. RRL, 2020, 4, 1900374.

37 B. E. Cohen, M. Wierzbowska and L. Etgar, Sustainable Energy \& Fuels, 2017, 1, 1935.

38 J. Hu, I. W. H. Oswald, H. Hu, S. J. Stuard, M. M. Nahid, L. Yan, Z. Chen, H. Ade, J. R. Neilson and W. You, ACS Mater. Lett., 2019, 1, 171.

39 J. Hu, I. W. H. Oswald, S. J. Stuard, M. M. Nahid, N. Zhou, O. F. Williams, Z. Guo, L. Yan, H. Hu, Z. Chen, X. Xiao, Y. Lin, Z. Yang, J. Huang, A. M. Moran, H. Ade, J. R. Neilson and W. You, Nat. Commun. 2019, 10, 1276.

40 J. Calabrese, N. L. Jones, R. L. Harlow, N. Herron, D. L. Thorn and Y. Wang, J. Am. Chem. Soc., 1991, 113, 2328.

41 G. C. Papavassiliou, G. A. Mousdis, C. P. Raptopoulou, A. Terzis and Z. Naturforsch. 1999, 54 b, 1405.

42 A. H. Slavney, R. W. Smaha, I. C. Smith, A. Jaffe, D. Umeyama and H. I. Karundasa, Inorg. Chem., 2017, 56, 46.

43 J. Hutter, M. Iannuzzi, F. Schiffmann and J. VandeVondele, Wiley Interdiscip. Rev. Comput. Mol. Sci, 2014, 4, 15.

44 S. Goedecker, M. Teter and J. Hutter, Phys. Rev. B, 1996, 54, 1703.

45 C. Hartwigsen, S. Goedecker and J. Hutter, Phys. Rev. B, 1998, 58, 3641.

46 M. Krack, Theor. Chem. Acc., 2005, 114, 145.

47 S. Grimme, J. Antony, S. Ehrlich and S. Krieg, J. Chem. Phys., 2010, 132, 154104.

48 S. Grimme, S. Ehrlich and L. Goerigk, J. Comp. Chem., 2011, $32,1456$.

49 J. VandeVondele and J. Hutter, J. Chem. Phys., 2007, 127, 114105. 